\title{
Balloon kyphoplasty for treatment of sacral insufficiency fractures
}

\author{
Report of three cases
}

\author{
H. Gordon DeEn, M.D., ANd Eric W. NotTMeier, M.D. \\ Department of Neurosurgery, Mayo Clinic, Jacksonville, Florida
}

\begin{abstract}
Sacral insufficiency fracture is a painful injury, for which no effective treatment currently exists. The objective of this study was to report on the clinical outcomes and technical aspects of balloon kyphoplasty, which was used in three patients with this injury.

Three elderly women with intractable pain from sacral insufficiency fractures were treated with polymethyl methacrylate (PMMA) injections into the sacrum by using a modified balloon kyphoplasty procedure. The visual analog scale pain score improved by four points in each case. Functional status was improved and analgesic medication requirements were decreased in all three patients. There were no complications associated with the procedure.

Because of the unique anatomy of the sacrum, it was difficult to monitor instrument placement and PMMA injection by using conventional fluoroscopy. BrainLAB image guidance was used in one case, and was helpful in guiding instrument placement and assuring accurate PMMA deposition at the fracture site.

Balloon kyphoplasty may be a treatment alternative in selected patients with sacral insufficiency fractures. BrainLAB image guidance may offer some advantages over conventional fluoroscopy with regard to the monitoring of instrument placement and PMMA injection.
\end{abstract}

KEY WORDS - balloon kyphoplasty - sacral insufficiency fracture

Sacral insufficiency fracture is a common and sometimes overlooked cause of debilitating low-back and sacral pain in elderly patients. ${ }^{5,7}$ The standard treatment involves prolonged periods of bed rest, which is often ineffective and leads to additional complications related to immobility.

Vertebral augmentation, in the form of vertebroplasty or kyphoplasty, has emerged as an effective treatment for vertebral compression fractures from the midthoracic region down to the L-5 level. ${ }^{12}$ Nevertheless, experience with these methods in the sacrum has been limited. We report our experience with three patients in whom sacral insufficiency fractures were treated with a modified balloon kyphoplasty procedure.

\section{CASE REPORTS}

We conducted an internal review board-approved retrospective study of three patients who underwent balloon kyphoplasty for treatment of sacral insufficiency fractures. Balloons and other instruments used for the kyphoplasty procedure were supplied by Kyphon, Inc. (Sunnyvale, CA).

\footnotetext{
Abbreviations used in this paper: $\mathrm{AP}=$ anteroposterior; $\mathrm{CT}=$ computerized tomography; $\mathrm{MR}=$ magnetic resonance; $\mathrm{PMMA}=$ polymethyl methacrylate; VAS = visual analog scale.
}

\section{Case 1}

History and Examination. This 72-year-old woman presented with a 6-week history of severe low-back and sacral pain, which developed after a fall. Her VAS pain scale score was 10 . The pain was activity limiting, and failed to respond to bed rest and hydromorphone. Bone mineral analysis showed osteopenia of the spine. Bilateral sacral insufficiency fractures were demonstrated on MR imaging of the lumbar spine and pelvis (Fig. 1).

Operation. Balloon kyphoplasty of the upper sacrum was performed with the aid of conventional fluoroscopic imaging. Balloon and PMMA volumes were $6 \mathrm{~cm}^{3}$ on the left and $4.5 \mathrm{~cm}^{3}$ on the right, and the maximum balloon pressure was 100 psi. There were no complications associated with the procedure. Postprocedure x-ray films of the pelvis showed PMMA deposition in the alae of the sacrum bilaterally (Fig. 2).

Postoperative Course. The postprocedure VAS pain score was 6 . The patient's functional status has improved, and her narcotic analgesic medication requirements have been reduced. This clinical improvement has been maintained, and no new fractures have occurred during 6 months of postprocedure follow-up care.

\section{Case 2}

History and Examination. This 75-year-old woman 


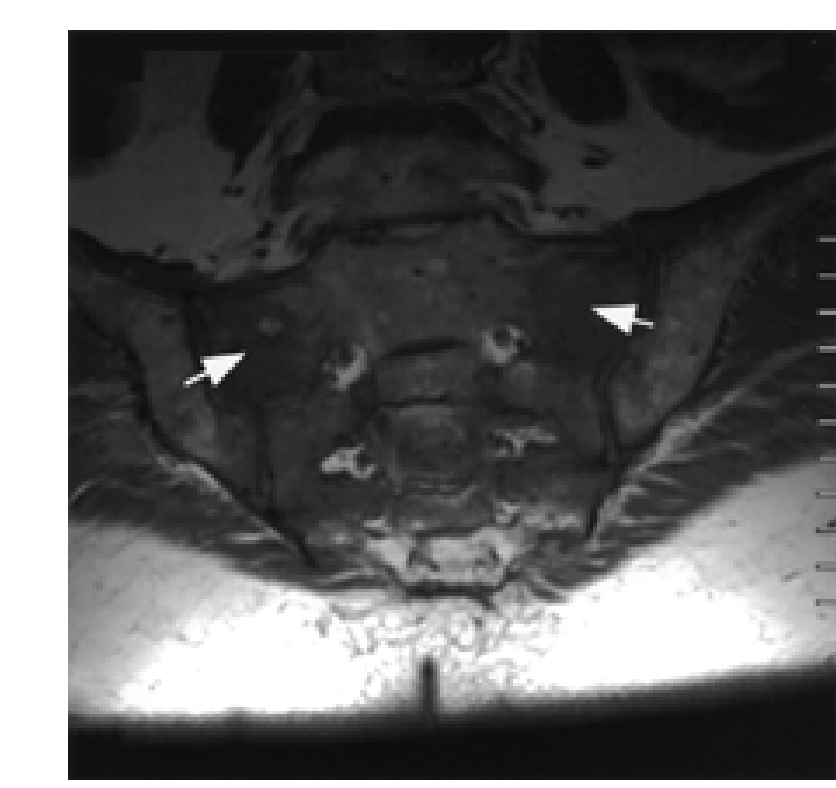

Fig. 1. Case 1. Coronal $\mathrm{T}_{1}$-weighted $\mathrm{MR}$ image demonstrating low signal intensity in the lateral aspect of the sacrum bilaterally (arrows), consistent with sacral insufficiency fracture.

with known osteoporosis presented with a 1-year history of severe, activity-limiting low-back and sacral pain, which was refractory to bed rest and hydrocodone. Her VAS pain score was 8. An MR imaging examination of the lumbar spine demonstrated a sacral insufficiency fracture.

Operation. Balloon kyphoplasty of the upper sacrum was performed with the aid of conventional fluoroscopic imaging. Balloon and PMMA volumes were $3 \mathrm{~cm}^{3}$ on each side, and the maximum balloon pressure was $100 \mathrm{psi}$. The patient tolerated the procedure well. Postprocedure $\mathrm{x}$ - ray films showed a small, clinically insignificant cement extravasation anterior to the sacrum.

Postoperative Course. The postprocedure VAS pain score was 4 . The patient's mobility has improved, and her narcotic analgesic medication requirements have decreased. This clinical improvement has been maintained, and no new fractures have occurred during 9 months of postprocedure follow-up care.

\section{Case 3}

History and Examination. This 78-year-old woman had a 1-year history of severe sacral and medial buttock pain that was unresponsive to bed rest and narcotic drugs, including morphine and hydrocodone. Her VAS pain score was 9 . She had a history of osteoporosis, previous lumbosacral fusion with instrumentation, and pelvic radiotherapy for carcinoma of the cervix. A CT scan of the lumbosacral region demonstrated complex sacral insufficiency fractures (Fig. 3).

Operation. Kyphoplasty of the sacrum was performed. Balloon and PMMA volumes were $4.5 \mathrm{~cm}^{3}$ on each side, and the maximum balloon pressure was 200 psi. A bone biopsy specimen was negative for malignancy.

Imaging Guidance. Because we had encountered some difficulty with the first two patients in verifying the cannula position in the sacrum while using standard fluoroscopy, we elected to use BrainLAB image guidance (BrainLAB, Chicago, IL) for the third patient. Because small incisions are used in this procedure, it is not possible to register the sacrum for image guidance by using a point- or surface-matching technique. The "CT-Fluoro merge" is a registration option on the BrainLAB system; this allows the spine to be registered to a preoperative CT scan by using a single AP and lateral fluoroscopic image. This obviates the need to take registration points manually on the dorsal aspect of the spine. Excellent registration

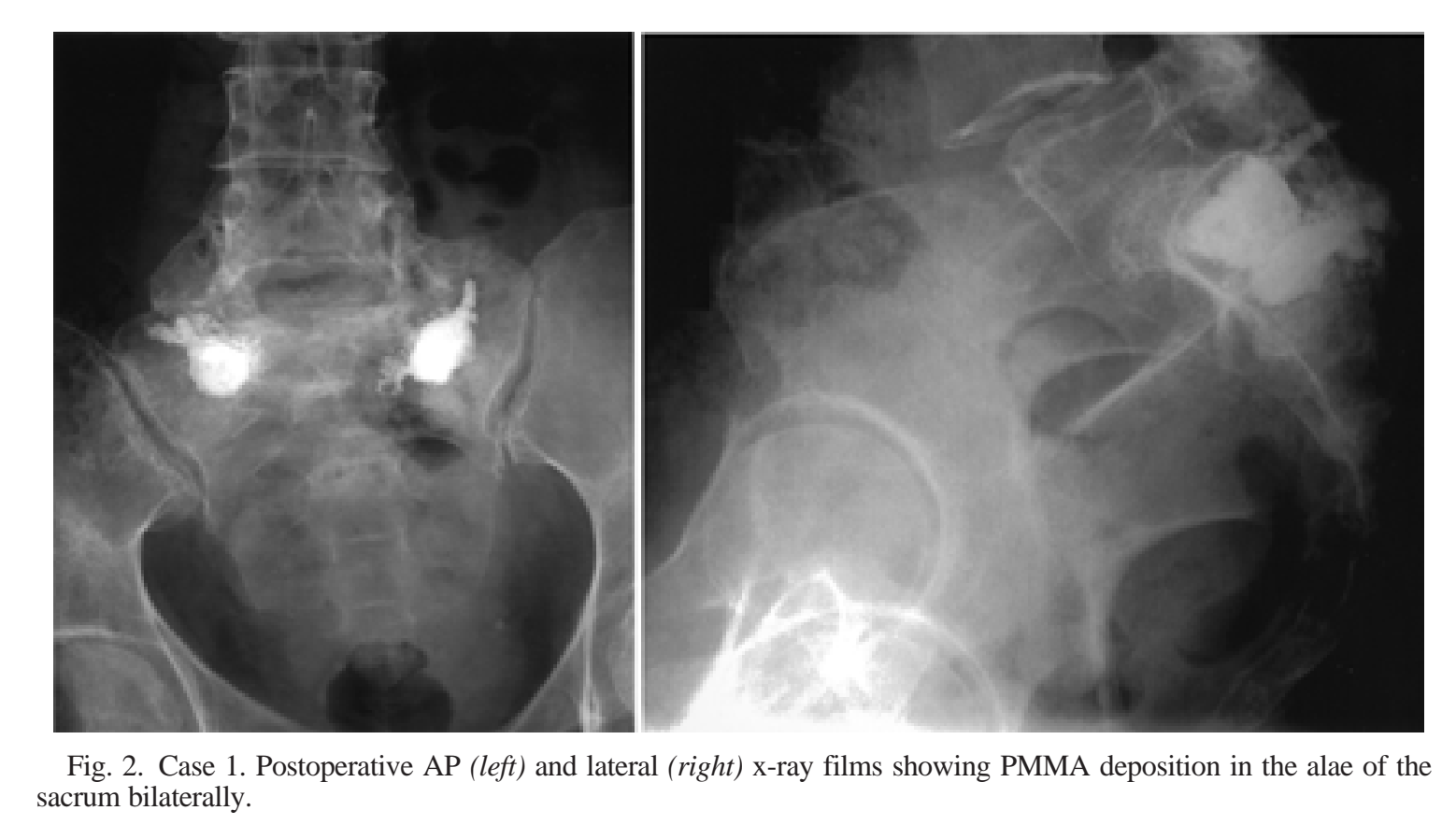




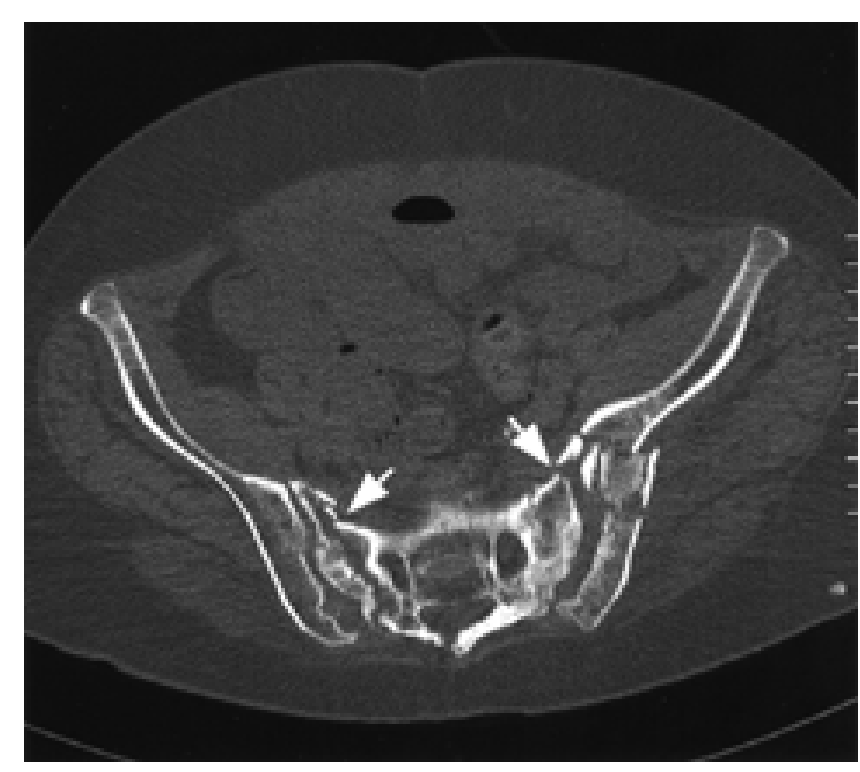

Fig. 3. Case 3. Axial CT scan of the sacrum demonstrating complex sacral insufficiency fractures (arrows).

was obtained in this case by using the CT-Fluoro merge option, which allowed the surgeon to navigate the sacrum accurately in three dimensions by using the preoperative CT scan. Accordingly, very precise cannula placement was confirmed, and PMMA injections were delivered directly into the fracture sites (Fig. 4).

Postoperative Course. At her 3-month follow-up visit, the patient's VAS pain score had decreased to 5, and her narcotic medication requirements were decreased. Postprocedure X-ray films of the pelvis showed PMMA deposition in the alae of the sacrum bilaterally (Fig. 5).

\section{DISCUSSION}

\section{Vertebral Augmentation}

Osteoporotic vertebral compression fracture is a common disorder that affects 700,000 patients per year in the US and causes significant pain and morbidity. ${ }^{11}$ Over the last decade, vertebroplasty and, more recently, kyphoplasty have become established as effective treatments for these fractures, with success rates of $95 \%$ being reported with either procedure. ${ }^{4}$ The technical aspects of vertebroplasty and kyphoplasty have been well described.,.6 Vertebral augmentation procedures have been used successfully for fractures in the thoracic and lumbar regions, from the midthoracic spine down to the L-5 level.

\section{Sacral Insufficiency Fracture}

Sacral insufficiency fractures are a relatively common but sometimes unrecognized cause of low-back pain in the elderly population. ${ }^{1}$ Osteoporosis is the leading cause, and older women are at particular risk. Other risk factors include chronic use of steroid drugs, inflammatory arthritis, radiation exposure, and previous lumbar fusion surgery. These fractures may be precipitated by minimal trauma. ${ }^{10}$

The diagnosis of sacral insufficiency fracture is some- times elusive on plain x-ray films. More advanced imaging modalities, including CT scanning, MR imaging, and radionuclide bone scans are often needed to confirm the diagnosis. The characteristic location for these fractures is in the lateral portion of the sacrum, parallel to the sacroiliac joint. ${ }^{7}$ A bone scan will reveal an $\mathrm{H}$-shaped pattern of increased uptake. ${ }^{5}$

Similar to treatment for osteoporotic fractures of the thoracic and lumbar spine, management of sacral insufficiency fractures has traditionally included periods of bed rest and a regimen of narcotic analgesic medications. These measures often fail to provide adequate pain relief, and may lead to complications associated with prolonged bed rest in elderly patients, including deep venous thrombosis, pulmonary embolus, pneumonia, and deconditioning, among others.

\section{Sacral Augmentation Procedures}

A safe, minimally invasive procedure that could provide pain relief and allow for resumption of normal physical activities would be of great benefit in this patient population. There have been isolated reports of vertebroplasty of the sacrum, so-called "sacroplasty," which has been used for both benign ${ }^{3,8,10}$ and metastatic ${ }^{2,9}$ lesions, with good clinical results. The rationale for this approach is the concept that sacral insufficiency fractures result from transmission of weight bearing, or axial loading, into the sacrum. ${ }^{7}$ The application of PMMA across the fracture could provide mechanical stabilization and prevent painful micromotion.

To our knowledge, balloon kyphoplasty of the sacrum has not been previously reported. Technically, there were some differences between a kyphoplasty of a vertebral body and a sacral kyphoplasty. It was more difficult to locate the curved ventral margin of the sacrum than the anterior cortex of a vertebral body with lateral fluoroscopy. Some investigators have recommended that venography be performed to confirm that cannula placement has not breached the anterior sacral cortex, ${ }^{3}$ although others have not found venography to be useful. ${ }^{10}$

Another key difference is that the cancellous bone in the sacrum is much softer and less dense than in a vertebral body, resulting in limited tactile feedback to the surgeon during cannula placement. Balloon pressures were generally low, and there was very little resistance to PMMA injection. The combination of difficulty with fluoroscopic imaging and reduced tactile feedback made it hard to tell whether PMMA might be extruding into the soft tissues of the buttock or pelvis during injection.

For these reasons, we elected to use BrainLAB image guidance for the third patient, and found that this modality significantly improved the accuracy of cannula placement and PMMA delivery. With the BrainLAB system, it was possible to navigate instruments and inject PMMA directly into the fractures identified on the preoperative CT scan. Although further work will be needed, BrainLAB image guidance may offer some advantages over conventional fluoroscopy with respect to instrument placement and PMMA delivery to the fracture site.

A potential concern with any method of sacral augmentation is medial migration of cement, which would compromise the exiting sacral nerve roots and sacral spinal 


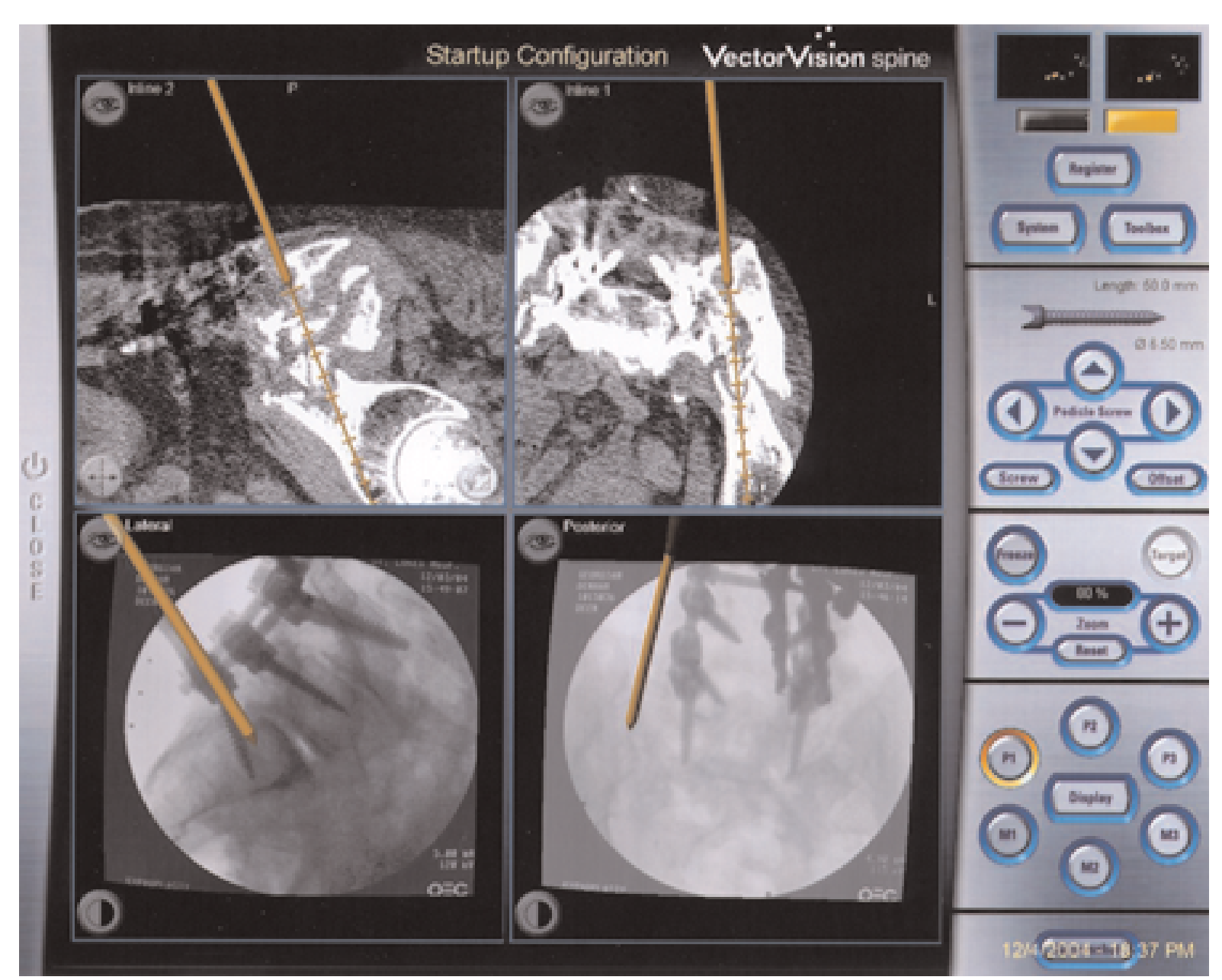

Fig. 4. Case 3. Representative real-time BrainLAB images used for navigation.

canal. We did not observe this complication in any of our cases.

It remains to be determined whether kyphoplasty is in any way superior to vertebroplasty or "sacroplasty" per-

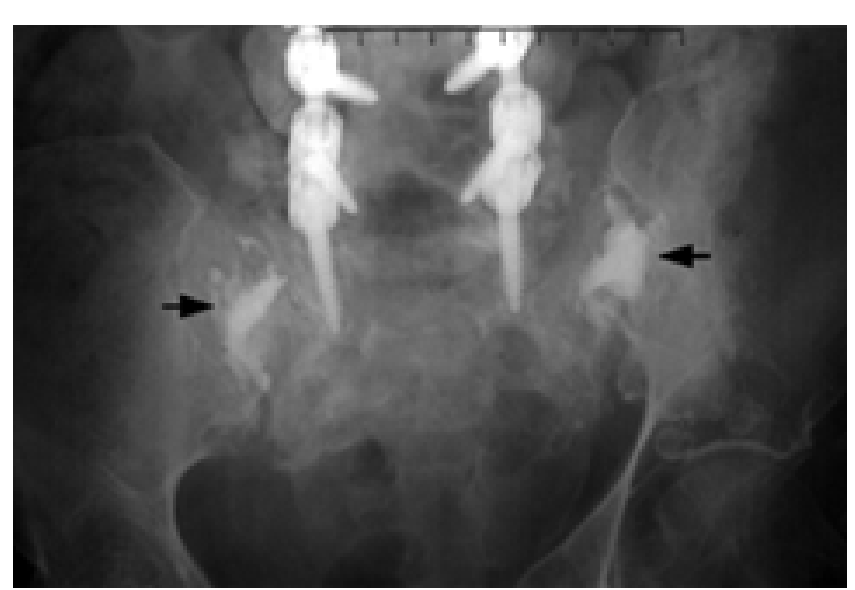

Fig. 5. Case 3. Postoperative AP x-ray film of the pelvis showing PMMA deposition in the alae of the sacrum bilaterally (arrows). formed without a balloon. It is unlikely that any meaningful degree of height restoration or fracture reduction can be achieved in these cases; however, the balloon inflation may be useful in compacting bone at the periphery of the fracture to reduce the incidence of cement extravasation.

\section{CONCLUSIONS}

Experience with these three patients indicates that balloon kyphoplasty of the sacrum is technically feasible and can provide good pain relief in individuals with sacral insufficiency fractures. BrainLAB image guidance may offer some advantages over conventional fluoroscopy with regard to the monitoring of instrument placement and PMMA injections. Further work is needed to assess long-term outcomes, to identify the best imaging method for monitoring the procedure, and to determine optimal PMMA volumes.

\section{Disclaimer}

Neither of the authors has a financial relationship with Kyphon, Incorporated.

\section{References}

1. Dasgupta B, Shah N, Brown H, et al: Sacral insufficiency frac- 


\section{Balloon kyphoplasty for sacral insufficiency fractures}

tures: an unsuspected cause of low back pain. Br J Rheumatol 37:789-793, 1998

2. Dehdashti AR, Martin JB, Jean B, et al: PMMA cementoplasty in symptomatic metastatic lesions of the $\mathrm{S} 1$ vertebral body. Cardiovasc Intervent Radiol 23:235-237, 2000

3. Garant M: Sacroplasty: a new treatment for sacral insufficiency fracture. J Vasc Interv Radiol 13:1265-1267, 2002

4. Garfin SR, Yuan HA, Reiley MA: New technologies in spine: kyphoplasty and vertebroplasty for the treatment of painful osteoporotic compression fractures. Spine 26:1511-1515, 2001

5. Grasland A, Pouchot J, Mathieu A, et al: Sacral insufficiency fractures: an easily overlooked cause of back pain in elderly women. Arch Intern Med 156:668-674, 1996

6. Lane JM, Johnson CE, Khan SN, et al: Minimally invasive options for the treatment of osteoporotic vertebral compression fractures. Orthop Clin North Am 33:431-438, viii, 2002

7. Leroux JL, Denat B, Thomas E, et al: Sacral insufficiency fractures presenting as acute low-back pain. Biomechanical aspects. Spine 18:2502-2506, 1993
8. Manfrè L: Vertebroplasty. Intervent Neuroradiol 9 (Suppl 2): 63-65, 2003

9. Marcy PY, Palussiere J, Descamps B, et al: Percutaneous cementoplasty for pelvic bone metastasis. Support Care Cancer 8:500-503, 2000

10. Pommersheim W, Huang-Hellinger F, Baker M, et al: Sacroplasty: a treatment for sacral insufficiency fractures. AJNR 24: 1003-1007, 2003

11. Riggs BL, Melton LJ III: The worldwide problem of osteoporosis: insights afforded by epidemiology. Bone 17 (Suppl 5): 505S-511S, 1995

12. Truumees E, Hilibrand A, Vaccaro AR: Percutaneous vertebral augmentation. Spine J 4:218-229, 2004

Manuscript received December 20, 2004.

Accepted in final form February 9, 2005.

Address reprint requests to: H. Gordon Deen, M.D., Department of Neurosurgery, 4500 San Pablo Road, Jacksonville, Florida 32224. email: hdeen@mayo.edu. 